PROCEEDINGS OF THE

AMERICAN MATHEMATICAL SOCIETY

Volume 136, Number 9, September 2008, Pages 3195-3204

S 0002-9939(08)09279-4

Article electronically published on May 2, 2008

\title{
TRIGONOMETRIC AND RADEMACHER MEASURES OF NOWHERE FINITE VARIATION
}

\author{
R. ANANTHARAMAN
}

(Communicated by N. Tomczak-Jaegermann)

Dedicated to my teacher, Krishna M. Garg

\begin{abstract}
Let $X$ be an infinite dimensional real Banach space. It was proved by E. Thomas and soon thereafter by L. Janicka and N. J. Kalton that there always exists a measure $\mu$ into $X$ with relatively norm-compact range such that its variation measure assumes the value $\infty$ on every non-null set. Such measures have been called "measures of nowhere finite variation" by K. M. Garg and the author, who as well as L. Drewnowski and Z. Lipecki have done related investigations. We give some "concrete" examples of such $\mu$ 's in the $l^{p}$ spaces defined using the (real) trigonometric system $\left(t_{n}\right)$ and the Rademacher system $\left(r_{n}\right)$ illustrating similarities and some differences. We also look at the extensibility of the integration map of these $\mu$ 's. As an application of the trigonometric example, we have the probably known result: For every $p \geq 1$, the function $\left(\Sigma\left(\left|t_{n}(t)\right|^{p}\right) / n\right)$ is unbounded on every set $A$ with positive measure.
\end{abstract}

\section{INTRODUCTION}

Let $(S, \Sigma, \lambda)$ be the measure space of the unit interval and $X$ be an infinite dimensional real Banach space with dual $X^{*}$. Let $\operatorname{ca}(\lambda):=\operatorname{ca}(S, \Sigma, \lambda, X)=$ ca denote the Banach space of all countably additive (c.a.) measures $\mu: \Sigma \rightarrow X$ with $\mu \ll \lambda$ endowed with the semi-variation norm $\|\mu\|:=\sup \{\|\mu(E)\|: E \in \Sigma\}$. Also let $\operatorname{cca}(\lambda):=\operatorname{cca}(S, \Sigma, \lambda, X)=$ cca. Denote the (closed) subspace of ca $(\lambda)$ consisting of measures with relatively norm compact ranges.

It was proved in [T] and a little later in $[\mathrm{J}-\mathrm{K}$ that in such an $X$ there is always a measure $\mu$ in $\operatorname{cca}(\lambda)$ such that its variation measure $|\mu|(\cdot)$ assumes the value infinity on every non-null set. As in $\mathrm{A}-\mathrm{G}$ ] let us call such measures (in both spaces) "of nowhere finite variation" (NWFV). These are called "everywhere of infinite variation" in Dr-L who with $\mathrm{A}-\mathrm{G}$, prove that such measures of NWFV form a residual set in the Banach spaces ca $(\lambda)$ and cca $(\lambda)$. In Dr-L there are several results about such measures. Both $[\mathrm{J}-\mathrm{K}]$ and $[\mathrm{Dr}-\mathrm{L}]$ ingeniously use the DvoretzkyRogers lemma [L-T, D-J-T] to produce a sequence $\left(\mu_{n}\right)$ of simple measures with small semi-variations and "uniformly large" variations; their sum is of NWFV (see also $[\underline{\mathrm{R}})$.

In this paper we give concrete examples of such measures (of NWFV) in the classical spaces $l^{p}$. Our method, different from above (used in [A-D]), is inspired by

Received by the editors April 10, 2007 and, in revised form, July 17, 2007.

2000 Mathematics Subject Classification. Primary 46G10; Secondary 28 B45.

(C) 2008 American Mathematical Society 3195

Reverts to public domain 28 years from publication 
$\mathrm{K}-\mathrm{K}$ where it is proved that there is a measure $\mu: \Sigma \rightarrow l^{2}$ whose range contains an interior point. To write a series for such measures we use OrthoNormal (ON) systems, especially the trigonometric system $\left(t_{n}\right)$ and the Rademacher system $\left(r_{n}\right)$ both on $[0,1]$. We are also interested in the domain of the integration operator $T$ associated with $\mu$; in general $T$ is only defined on $L^{\infty}(\lambda)$. The paper A1 considers the $\mu$ 's whose $T$ 's do not extend to any $L^{p}$ for finite $p$; the question regarding extension to no $L^{p}\left(\lambda_{A}\right)$ is left open.

Section 2 contains a summary of the concepts and results we use. We state fundamental results due to Bartle, Dunford and Schwartz in Proposition 2.1. Next we state an extension of a theorem from $\mathrm{A}-\mathrm{D}$ ] that allows us to write measures in $X$ using $l_{\text {weak }}^{2}(X)$ sequences in $X$ "against" ON systems in $L^{2}$; we call these measures "Trig" or "Rademacher" depending on the ON system used. Let us emphasize that the Trig system $\left(t_{n}\right)$ uses real scalars.

We consider Trig measures in Section 3 and Rademacher ones in Section 4. In Section 3 we first use the sequence $\left(e_{n} / n^{1 / p}\right)$ in $l^{p}$ to define the Trig measure into $l^{p}$ for (all $p \geq 1$ ) and prove in Theorem 3.1 that this measure $\mu$ is in cca $(\lambda)$ and of NWFV. Also the baclco(range), or balanced closed convex hull, of the range of a suitable multiple of $\mu$ contains the above sequence. In Corollary 3.3 we have the probable known result: the (measurable) function $\left(\Sigma\left(\left|t_{n}(t)\right|^{p}\right) / n\right)$ is unbounded on every set $A$ with $\lambda(A)>0$. In Proposition 3.4 we consider the extension properties of the integration map of this measure, which are better than $L^{2}$ stated in Proposition 2.6. Next we consider the sequence $\left(e_{n}\right)$ (i.e., without the weight $1 / n^{1 / p}$ ) in $l^{p}$ with the trigonometric system to get an example of NWFV (only in) ca $(\lambda)$ in Proposition 3.5 but now $p \geq 2$ and the map $T$ of this example also does better than $L^{2}$.

Analogously to Theorem 3.1, we consider the sequence $\left(e_{n} / n^{1 / p}\right)$ in $l^{p}$ (all $p \geq 1$ ) with the Rademacher sequence in Proposition 4.1 to get another example of NWFV. Next we consider the analog of Proposition 3.5 for the Rademacher measure in Proposition 4.5, Our tools of proof are also from Fourier series [Z].

Let us note in passing a difference between these measures. Due to the completeness of the trigonometric system, the trigonometric measure $\mu$ is an injective measure $[\mathrm{K}-\mathrm{K}]$. In contrast, the Rademacher sequence is incomplete on every set $A \in \Sigma[\mathrm{K}-\mathrm{S}]$, and it is a Liapunov measure $[\mathrm{K}-\mathrm{K}]$;

In Proposition 4.3 we prove that the sequence $\left(e_{n} / n^{1 / 2 p}\right)$ in $l^{p}($ all $p>1)$ lies in baclco(range) of a measure of NWFV. Curiously, this contrasts with a result in A-D that the sequence $\left(e_{n} / n^{1 / 2}\right)$ in $l^{1}$ does not lie in baclco(range) of any measure.

In Proposition 5.1 we use an elegant result from [P-Ro] characterizing membership in $\operatorname{cca}(\lambda)$ to show that if $\mu$ is of NWFV in cca $(\lambda)$, then there is a measure $\nu$ of sigma finite variation such that $K_{\mu} \subset K_{\nu}$. Hence, we answer in the negative a question in [A-D]: Must the sequence $\left(e_{n} / n\right)$ in $l^{1}$ always lie in range of a measure with non- $\sigma$ finite variation?

Problems. In addition to the one on (non)extension of the map $T$, we do not know if the examples in this paper can be done in an abstract context.

\section{Preliminaries on measures defined by ON systems}

For vector measures concepts and results, we follow $[\mathrm{Du}-\mathrm{S}$, $\mathrm{D}-\mathrm{U}, \mathrm{K}-\mathrm{K}$, and for Banach spaces [L-T, [D-J-T]. The control measure $\lambda$ in Proposition 2.1, due to Bartle, Dunford and Schwartz, enables integration of scalar functions in $L^{\infty}(\lambda)$ with 
respect to $\mu$. Following Lindenstrauss $\mathrm{L}$ in his elegant proof of Liapunov's theorem, we denote this operator by $T$; i.e., $T(f)=\int_{S} f d \mu:=\int f d \mu$. Furthermore, we use the weakly compact convex sets $K=\operatorname{clco} \mu(\Sigma)$ and $C:=C_{\mu}=$ baclco $\mu(\Sigma)=K-K$ (balanced closed convex hull of the range $\mu(\Sigma)$ ). We employ the symbol $\chi_{E}$ to denote the characteristic function of the set $E$.

Fundamental facts about an arbitrary measure and its integration operator $T$ are in

Proposition 2.1 ([Du-S], $[\mathrm{D}-\mathrm{U}],[\mathrm{K}-\mathrm{K}])$. Let $\mu: \Sigma \rightarrow X$ be countably additive.

(i) There always exists a finite positive measure $\lambda$ that controls $\mu$; i.e.,

$$
\|\mu\|(E) \rightarrow 0 \quad \text { iff } \lambda(E) \rightarrow 0 \text { for } E \text { in } \Sigma .
$$

(ii) The integration operator $T: L^{\infty}(\lambda) \rightarrow X$ is bounded and weak-* to weakly continuous.

(iii) With $P=\{\varphi: 0 \leq \varphi(t) \leq 1$ for $\lambda$ a.e. $t\}$, we have $T(P)=K_{\mu}=\operatorname{clco} \mu(\Sigma)$, which is weakly compact and convex.

So is $C_{\mu}:=C=K-K=T(B)$, where $B$ is equal to the closed unit ball in $L^{\infty}(\lambda)$.

(iv) The adjoint $T^{*}$ of $T$ maps $X^{*}$ into $L^{1}(\lambda)$.

Remark. We hope that the use of the same symbol " $C$ " to denote the set in (iii) above and a constant below will not cause confusion.

2.2. Bessel's inequality. Our examples depend on Bessel's inequality: For an arbitrary ON sequence $\left(f_{n}\right)$ in $L^{2}:=L^{2}[0,1]$ the sequence $\left(\left\langle g, f_{n}\right\rangle\right)$ is in $l^{2}$ for each $g$ in $L^{2}$ and $\left(\Sigma\left\langle g, f_{n}\right\rangle^{2}\right)^{1 / 2} \leq\|g\|_{L^{2}}$.

Our ON sequences. We consider orthogonal sequences $\left(f_{n}\right)$ in $L^{2}$ such that (i) $\left|f_{n}(t)\right| \leq 1$ for a.e. $t$ in $[0,1]$, and (ii) there exist positive finite numbers $a, b$ such that $a \leq\left\|f_{n}\right\|_{L^{2}} \leq b$ for all $n$. Let us call these ON.

We note that for the Bessel inequality to hold, it is sufficient if the number $b$ in condition (ii) does not exceed 1 (actually less than $\sqrt{2}$ will do) as can be seen by inspecting the proof of the classical statement above. The right side will change to $\leq C_{1}\|g\|_{L}^{2}$ for some finite positive $C_{1}\left(=\left(2-b^{2}\right)^{1 / 2}\right)$. Condition (i) is not needed to prove NWFV of our examples; it is only needed to put a sequence in the set $C_{\mu}$ (see Proposition 2.6 below).

2.3. The trigonometric or Trig system. This is denoted by $\left(t_{n}(t)\right)$ on $[0,1]$ and is with real scalars. More precisely, this sequence of functions is $\{1, \cos 2 \pi t$, $\sin 2 \pi t, \cos 4 \pi t, \sin 4 \pi t$, etc., $\}$ in this order. We use the Trig system as above; i.e., normalized in $L^{\infty}$.

2.4. The Rademacher sequence $\left(r_{n}(t)\right)$. This is defined by

$$
r_{n}(t)=\operatorname{sign} \sin \left(2^{n} \pi t\right) \text { for } 0 \leq t \leq 1
$$

As in $[\mathrm{A}-\mathrm{D}$, our method of writing measures is inspired by $[\mathrm{K}-\mathrm{K}]$. We use it as follows.

Definition 2.5. A sequence $\left(x_{n}\right)$ in $X$ is said to be in $l_{\text {weak }}^{2}(X)$ if the sequence $\left(x^{*}\left(x_{n}\right)\right)$ of scalars is in $l^{2}$ for each $x^{*}$ in $X^{*}$. There is a corresponding definition for $l_{\text {weak }}^{p}(X)$, but we mainly use $p=2$. 
Let us note that the unit vector basis $\left(e_{n}\right)$ of $X=l^{p}$ is in $l_{\text {weak }}^{2}(X)$ iff $p \geq 2$. If the sequence $\left(x_{n}\right)$ is in $l_{\text {weak }}^{2}(X)$, then a closed graph argument shows (e.g., D]) that $\sup \left\{\left[\Sigma x^{*}\left(x_{n}\right)^{2}\right]^{1 / 2}:\left\|x^{*}\right\| \leq 1\right\}=C$ finite.

In the next proposition only the Rademacher sequence was considered in $\mathrm{A}-\mathrm{D}$; the proof holds for any $\mathrm{ON}$ sequence. As our examples come from sequences in $l_{\text {weak }}^{2}(X)$, they are countably additive due to part(ii) in

Proposition 2.6 ( $\mathrm{A}-\mathrm{D}])$. Let $X$ be a real Banach space, $\left(x_{n}\right)$ be a sequence in $l_{\text {weak }}^{2}(X)$, and $\left(f_{n}(t)\right)$ any $O N$ sequence in $L^{2}$. Then

(i)

$$
\mu(E):=\Sigma\left(\int_{E} f_{n}(t) d t\right) x_{n} \quad \text { is in } X \text { for every } E \text { in } \Sigma,
$$

(ii) $\mu$ is countably additive and $\mu \ll \lambda$,

(iii) the integration operator $T$, extends to $L^{2}$ and is bounded, and

(iv) for some $C>0$ the baclco $C \mu(\Sigma)$ contains $\left(x_{n}\right)$.

The paper [A2] considers the sequence of Rademacher averages $\left(\int_{E} r_{n}(t) d t\right)$ of sets in $\Sigma$. It is proved that for "most sets" in $\Sigma$ in the sense of a Baire Category the sequence is not in $l^{p}$ for any $p<2$. The methods used there apply to Trig or ON systems in general. Hence the condition $p \geq 2$ is needed in Propositions 3.5 and 4.5. We note that the measures below defined into $c_{0}$ are of finite variation.

\section{The trigonometric MeAsures into $l^{p}$}

We note that with $X=l^{p}$, the sequence $\left(x_{n}\right):=\left(e_{n} / n^{1 / p}\right)$ is in $l_{\text {weak }}^{2}(X)$. Hence, by Proposition 2.6 equation (2) below defines countably additive measures $\mu: \Sigma \rightarrow l^{p}$ into all $l^{p}$,

$$
\text { Trig: } \quad \mu(E):=\Sigma\left(\int_{E} t_{n}(t) d t\right)\left(e_{n} / n^{1 / p}\right) .
$$

In Theorem 3.1 we need to apply a classical criterion ([Du-S Ch4). A set $K$ in $l^{p}$ is relatively norm-compact iff (a) $K$ is norm-bounded, and (b) we have $\lim \Sigma\left\{\left|a_{i}\right|^{p}: i \geq\right.$ $n\}=0$ as $n \rightarrow \infty$, uniformly for $a=\left(a_{i}\right)$ in $K$.

We also need the Riemann-Lebesgue lemma [Z]. The Fourier coefficients of a function in $L^{1}$ tend to zero as $n$ tends to infinity. We use the fact ([D-U]) that the $l^{p}$-spaces possess the Radon-Nikodym property (RNP) in

Theorem 3.1. Let $X=l^{p}$ for $p \geq 1$ and be the trigonometric measure defined by (2). Then $\mu$ is (i) in cca, (ii) of $N W F V$. Further, (iii) the baclco(range) of $C \mu$ contains the sequence $\left(e_{n} / n^{1 / p}\right)$ for some $C>0$.

Proof. (i) We need to verify conditions (a) and (b) in the criterion above. As for (a) in our case we know (from Proposition 2.1) that $K$ is weakly compact.

(b) Using (2), we need to verify that we have

$$
\left.\lim \Sigma\left\{(1 / i)\left(\left|\int_{E} t_{i}(t) d t\right|\right)^{p}\right): i \geq n\right\}=0
$$

as $n \rightarrow \infty$ uniformly for $E$ in $\Sigma$. 
Case 1: $p \geq 2$. We rewrite the sum in (3) as

$$
\begin{aligned}
& \Sigma\left\{\left(1 / i^{1 / p}\right)^{p}\left(\left|\int_{E} t_{i}(t) d t\right|\right)^{p}: i \geq n\right\} \quad \text { (we omit the " } i \geq n \text { " in the following) } \\
& \leq\left[\Sigma\left\{\left(1 / i^{1 / p}\right)^{2}\left(\left|\int_{E} t_{i}(t) d t\right|\right)^{2}\right\}\right]^{p / 2} \quad\left(\text { since } p \geq 2, \text { we have }\|x\|_{p} \leq\|x\|_{2}\right) \\
& \leq(1 / n)\left[\Sigma\left\{\left(\left|\int_{E} t_{i}(t) d t\right|\right)^{2}\right\}\right]^{p / 2}
\end{aligned}
$$

and the quantity within $\left\{[\cdots] \leq \lambda(E)^{2}\right.$ due to Bessel's inequality. Hence the sum in (3) is $\leq(1 / n)\left(\lambda(E)^{p}\right) \leq 1 / n$ which tends to zero independently of $E$ in $\Sigma$, as required.

Case 2: $1 \leq p<2$. Let $X=l^{p}$. In case $p=1$, then the conclusion that $\mu$ is in cca $(\lambda)$ follows from Proposition 2.1 and the Schur property that $l^{1}$ has. In case $1<p<2$, we may apply a theorem of Rosenthal $\mathrm{Ro}$ that every operator from $L^{\infty}$ into such $X$ is compact. However the conclusion can be proved independently and analogously to Case 1 with the help of Holder's inequality with $r=2 / p(>1)$ with $r^{*}$ its conjugate index.

(ii) Suppose that the variation $|\mu|(A)$ is finite and positive on $A$. With the help of the RNP of $l^{p}$ we obtain a Bochner integrable function $g: A \rightarrow l^{p}$ such that $\mu(E)=\int_{E} g(t) d \lambda(t)$ for all $E \in \Sigma, E \subset A$. So we have (from (2) )

$$
g(t)=\Sigma t_{n}(t) e_{n} / n^{1 / p} \text {, whence }\|g(t)\|^{p}=\Sigma\left|t_{n}(t)\right|^{p} / n \text {, also a.e. } t \text { in } A
$$

As $\|g(t)\|$ is in $L^{1}(\lambda)$ on $A$, there exists a subset $B$ of $A,(B \in \Sigma)$ with $\lambda(B)>0$ on which $\|g(t)\|$ is bounded. Let us write $A$ in place of $B$. For fixed $m$ we have

$$
\begin{aligned}
\left\|t_{m} \chi_{A}\right\|_{p} & =\left(\int_{A}\left|t_{m}(t)\right|^{p} d l \lambda(t)\right)^{1 / p} \\
& =\sup \left\{\left(t_{m}, f \chi_{A}\right): f \in L^{q}, \text { with }\|f\| \leq 1\right\},
\end{aligned}
$$

where $\left(t_{m}, f \chi_{A}\right)=\left(\int_{A} t_{m}(t) f(t) d \lambda(t)\right)$. Now $t_{m}=1$, or $\cos 2 \pi n t$, or $\sin 2 \pi n t$ and so $\|t m\|_{\infty} \leq 1$. Hence $t_{m}$ qualifies as an $f$ in (5) and we obtain

$$
\left\|t_{m}\right\|_{p} \geq\left(t_{m}, t_{m} \chi A\right) \quad \text { or } \quad \int_{A}\left|t_{m}(t)\right|^{p} d \lambda(t) \geq\left[\int_{A} t_{m}(t)^{2} d \lambda(t)\right]^{p} .
$$

We may assume that $m \geq 2$. Then $t_{m}=\cos 2 \pi m t$ or $\sin 2 \pi m t$. Using an elementary trigonometric formula, we get $t_{m}^{2}=\frac{1}{2}(1 \pm \cos 4 \pi m t)$, and so we have

$$
\int_{A} t_{m}^{2} d \lambda(t)=\frac{1}{2}(1 \pm \cos 4 \pi m t) d t
$$

Now, the second term in (77) tends to zero as $m$ tends to infinity, thanks to the Riemann-Lebesgue lemma. Thus for all sufficiently large enough $m$, (7) gives

$$
\int_{A} t_{m}^{2} d \lambda(t) \geq \frac{1}{4} \lambda(A)
$$

and so from (6) we get $\int_{A}\left|t_{n}(t)\right|^{p} d l(t) \geq C>0$ for all sufficiently large $m$.

Now let us integrate (4) as we may, to get the contradiction that a finite number $C_{1} \geq C \Sigma\left\{1 / n: n \geq n_{0}\right\}$. Hence $|\mu|(A)=\infty$ and $\mu$ is of NWFV. The above also works for $p=1$, upon replacing $L^{q}$ by $L^{\infty}$ and noting that $t_{n}$ is in $L^{\infty}(\lambda)$. Part (iii) follows from Proposition 2.6 since the trigonometric system satisfies both conditions (i) and (ii) in 2.2 or we can verify directly from (2) using the trigonometric identity in (6) that $T\left(t_{m}\right)=1 / 2\left(e_{n} / n^{1 / p}\right)$. Hence $C=2$ and so Theorem 3.1 is proved. 
Let us note from (4) in Case 2, part (ii) that $\|g(t)\| \notin L^{r}(A)$ for any $r>0$ (even $0<r<1$ ), for if it did, then $\|g(t)\|$ would be (essentially) bounded on some subset $B$ of $A$, which is false by the above. Hence we get

Corollary 3.2. The function $\left(\Sigma\left(\left|t_{n}(t)\right|^{p}\right) / n\right)$ is not in $L^{r}$ for any $r>0$ on every set $A$ with $\lambda(A)>0$.

Corollary 3.3. The function $\left(\Sigma\left(\left|t_{n}(t)\right|^{p}\right) / n\right)$ is unbounded on every set $A$ with $\lambda(A)>0$.

In the next result we consider the domain of the integration map $T$. We need another classical result, the Hausdorff-Young theorem [Z]. If $1<p<2, q$ is the index conjugate to $p$ and the function $f$ is in $L^{p}$, then the sequence $\left(f^{\wedge}(n)\right)$ of its Fourier coefficients is in $l^{q}$ and we have $\left(\Sigma\left|f^{\wedge}(n)\right|^{q}\right)^{1 / q} \leq\|f\|_{L}^{p}$. We see from Proposition 2.6 that for measures defined by $l_{\text {weak }}^{2}(X)$ sequences, the domain of the integration operator $T$ extends up to $L^{2}$ in general; it does slightly better as we see below.

Proposition 3.4. Let $\mu: \Sigma \rightarrow l^{p}$ be the trigonometric measure defined by (2).

(i) If $1<p<2$, then $T$ extends to $L^{p}$.

(ii) If $X=l^{1}$ or $l^{2}$, then $T$ extends to $L^{p}$ for all $p>1$.

(iii) If $p>2$, then $T$ extends to $L^{q}$, where $q$ is the index conjugate to $p$.

Proof. (i) Let $1<p<2$ and $\mu: \Sigma \rightarrow X=l^{p}$. We need to show that $T$ maps $L^{p}$ into $X$ and is bounded. For this, as $L^{\infty}$ is dense in $L^{p}$, it is enough to show that

$$
\|T(f)\| C\|f\|_{L}^{p} \quad \text { for } f \text { in } L^{\infty} .
$$

We have from (2)

$$
T(f)=\Sigma\left(\int f t_{n}(t) d t\right)\left(e_{n} / n^{1 / p}\right) .
$$

Let $x^{*}=\left(x_{n}^{\prime}\right)$ in $X^{*}=l^{q}$. We get $x^{*} o T(f)=\Sigma\left(\int f t_{n}(t) d t\right)\left(x_{n}^{\prime} / n^{1 / p}\right)$, and so by Holder's inequality

$$
\begin{aligned}
\left|x^{*} o T(f)\right| & \leq\left[\Sigma \mid\left(\int f t_{n}(t) d t \mid\right)^{q}\right]^{1 / q}\left[\Sigma\left|x_{n}^{\prime}\right|^{p} / n\right]^{1 / p} \\
& \leq\|f\|_{L}^{p}\left[\Sigma\left|x_{n}^{\prime}\right|^{p} / n\right]^{1 / p}
\end{aligned}
$$

thanks to the Hausdorff-Young theorem.

Since we have $1<p<2<q$, we see that $r=q / p>1$. The Holder inequality applied to the sum within [...] in (9) gives

$$
\left|x^{*} o T(f)\right| \leq\|f\|_{L}^{p}\left(\Sigma ( | x _ { n } ^ { \prime } | ^ { q } ) ^ { 1 / q } \left(\Sigma\left(1 / n^{r^{*}}\right)^{1 / p r^{*}},\right.\right.
$$

where $r^{*}$ is an index conjugate to $r$ and as $r^{*}>1$ the series in the last $(\ldots)$ converges to a finite sum. We have $\left|x^{*} o T(f)\right| \leq C\|f\|_{L}^{p}\left\|x^{*}\right\|$ for every $x^{*}$ with $\left\|x^{*}\right\| \leq 1$, and so $\|T(f)\| \leq C\|\mid f\|_{L}^{p}$, which by (8) suffices to prove (i).

(ii) Let us prove this for $\mu: \Sigma \rightarrow l^{2}$; the argument for the case of $l^{1}$ is similar. It is sufficient to show that $T$ extends to $L^{p}$ for $1<p<2$, since the extension of $T$ to $L^{p}$ for $p \geq 2$ follows due to the inclusion between $L^{p}$ spaces and monotonicity of norms. We have from (8⿴囗十) that $\|T(f)\|_{2}=\left[\Sigma\left|\int f t_{n}(t) d t\right|^{2}(1 / n)\right]^{1 / 2}$. Now, as 
$p<2$, we have $q>2$ so that we may let $r=(q / 2)>1$ and $r^{*}>1$ as before. We have by Holder's inequality

$$
\|T(f)\|_{2} \leq\left[\Sigma\left|\int f t_{n}(t) d t\right|^{q}\right]^{1 / q}\left[\Sigma(1 / n)^{r^{*}}\right]^{1 / 2 r^{*}} \leq C\|f\|_{L}^{p},
$$

thanks again to the Hausdorff-Young theorem due to $1<p<2$.

(iii) The proof is similar upon applying the Hausdorff-Young theorem; hence Proposition 3.4 is proved.

Trigonometric measures in ca and into $l^{p}$ with $p \geq 2$. The trigonometric measure $\mu$ can be defined without the weight $\left(1 / n^{1 / p}\right)$. Now $\mu$ takes values in $X=l^{p}$ with $p \geq 2$ and is countably additive thanks to Proposition 2.6 since $\left(e_{n}\right)$ is in $l_{\text {weak }}^{2}(X)$ for such $p$. The need for the condition $p \geq 2$ (in [A2]) is mentioned after Proposition 2.6. We redefine

$$
\text { Trig : } \quad \mu(E):=\Sigma\left(\int_{E} t_{n}(t) d t\right)\left(e_{n}\right) .
$$

This measure has properties analogous to those in Theorem 3.1. Most of the properties are proved as in Theorem 3.1.

Proposition 3.5. Let $p \geq 2$ and $\mu: \Sigma \rightarrow l^{p}$ be defined by (10). Then

(i) its $T$ extends to $L^{q}$,

(ii) $\mu$ is $N W F V$,

(iii) the set $K_{\mu}=\operatorname{clco} \mu(\Sigma)$ is (only) weakly compact, and

(iv) has no interior points.

Proof. (i) This follows from the Hausdorff-Young theorem as in Proposition 3.4. part (i).

(ii) This is similar to part (iii) in Theorem 3.1

(iii) As $K_{\mu} \subset C_{\mu}$ (see Proposition [2.1), it is enough to prove that $C_{\mu}$ is not norm-compact. But then $\left|t_{n}\right| \leq 1$ and so $T\left(t_{n}\right) \in C_{\mu}$ (by Proposition 2.1).

Now from (10) we get

$$
T\left(t_{n}\right)=\left(\int_{E} t_{n}^{2} d t\right) e_{n}=C_{n} e_{n}
$$

where $C_{\mu}=1$ if $n=1$ and $\frac{1}{2}$ if $n>1$, as we see by using the elementary trigonometric formula used in the proof of (ii) of Theorem 3.1. Hence the set $C_{\mu}$ contains the unit vectors $1 / 2\left(e_{n}\right)$, and thus it is not norm-compact.

(iv) Suppose that $K$ has an interior point (i.e., in norm). As $K_{\mu} \subset C_{\mu}$, the latter set has an interior point too. But then as $C=C_{\mu}=-C_{\mu}$, we see that zero is an interior point of $C_{\mu}$; i.e., there is $r>0$ such that the closed ball $B(0, r) \subset C$. We have from Proposition 2.1. $C=T(B)$, where $B=$ closed unit ball of $L^{\infty}$. Hence we obtain,

$$
T\left(L^{\infty}\right)=T(\{\bigcup n B: n \geq 1\})=\bigcup T(n B)=\bigcup n T(B)=\bigcup n C=Y \text {, say. }
$$

As $B(0, r) \subset C$, we have $Y \supset \bigcup\{n B(0, r): n \geq 1\}=l^{p}$, and so $T: L^{\infty} \rightarrow l^{p}$ is onto, and we apply the Fourier uniqueness theorem to conclude that $L^{\infty}$ and $l^{p}$ are isomorphic, a contradiction. Hence $K$ has no interior points as claimed. 


\section{RADEMACHER MEASURES INTO $l^{p}$}

The Rademacher sequence $\left(r_{n}(t)\right)$ is defined in subsection 2.4. We define

$$
\text { Rademacher : } \quad \mu(E):=\Sigma\left(\int_{E} r_{n}(t) d t\right)\left(e_{n} / n^{1 / p}\right) \text {. }
$$

As we saw in the case of the trigonometric measure (defined by (2)), this too has its values in $l^{p}$ (for $p \geq 1$ ) and is countably additive. Let us call this the "Rademacher measure". We recall Khintchine's inequality [D], D-J-T]: If the sequence $\left(\alpha_{n}\right)$ is in $l^{2}$, then the function $f:=\Sigma \alpha_{n} r_{n}$ is in $L^{p}$ for all $p \geq 1$.

Part (i) of Proposition 4.1] is in [A1] but, in the context of ca (see (14) below), it contrasts with Proposition 3.4

Proposition 4.1. Let $X=l^{p}$ with $p \geq 1$. The Rademacher measure $\mu$ in (11) has the following properties:

(i) Its $T$ extends to $L^{r}$ for all finite $r>1$ but not to $L^{1}(A)$ for any $A$ with $\lambda(A)>0$. Furthermore, $\mu$ is (ii) in cca and of (iii) $N W F V$.

Proof. (i) We have $T: L^{\infty} \rightarrow X$ and as we see from (11),

$$
T(f)=\Sigma\left(\int f r_{n}(t) d t\right) e_{n} / n^{1 / p}
$$

for each $f$ in $L^{\infty}$. The adjoint $T^{*}: X^{*} \rightarrow L^{1}$ (e.g., [D-U]) and for any $x^{*}=\left(x_{n}^{\prime}\right)$ in $X^{*}=l^{q}$, we obtain $T^{*}\left(x^{*}\right)=\Sigma x_{n}^{\prime} r_{n} / n^{1 / p}$.

We claim that the sequence $\left(x_{n}^{\prime} / n^{1 / p}\right)$ of coefficients is, for all $p$, in $l^{2}$ and then $T^{*} x^{*}$ will be in $L^{r}$ for any $r>1$ thanks to Khintchine's inequality. Hence $T^{*}$ maps into $L^{r}$ for any $r>1$ and so $T$ maps from any $L^{r}$.

We need to verify our claim. In the case $p \geq 2$, we have $q \leq 2$. Now $\left|x_{n}^{\prime} / n^{1 / p}\right| \leq$ $\left|x_{n}^{\prime}\right|$ for all $n$, and $x^{*}$ is in $l^{q} \subset l^{2}$, thus the sequence $\left(x_{n}^{\prime} / n^{1 / p}\right)$ is in $l^{2}$ as claimed. If $p<2$ we note that the sequence $\left(1 / n^{1 / p}\right)$ is in $l^{2}$ and that $\left(x_{n}^{\prime}\right)$ is bounded. Hence we have the claim for all $p$ and part(i). To see that $T$ cannot extend to $L^{1}(A)$ we can use part (iii) (without circularity); for if it did, $\mu$ will have (e.g., D-U]) finite variation on (subsets of ) $A$, which contradicts (iii) below.

(ii) The proof uses the classical criterion in Du-S and is similar to Theorem 3.1,

(iii) The proof is similar to the one in Theorem 3.1 using RNP of $l^{p}$. The fact that $\left|r_{n}(t)\right|=1$ makes the proof easier. Hence Proposition 4.1 is proved.

Remark 4.2. It is shown in [A-D] that the sequence $\left(e_{n} / n^{1 / 2}\right)$ in $l^{1}$ does not lie in baclco (range) of any measure (it is not in $\left.l_{\text {weak }}^{2}\left(l^{1}\right)\right)$. The contrast with $p>1$ in the next proposition seems curious.

Proposition 4.3. Let $X=l^{p}$ for $p>1$.

(i) For each $E$ in $\Sigma, \mu(E):=\Sigma\left(\int_{E} t_{n}(t) d t\right)\left(e_{n} / n^{1 / 2 p}\right)$ is in $X, \mu$ is (ii) in cca and (iii) of $N W F V$. The sequence $\left(e_{n} / n^{1 / 2 p}\right)$ is in baclco(range) of a constant multiple of $\mu$.

Proof. Let us only prove (i) as part (ii) is similar to that in Theorem 3.1 and (iii) follows from Proposition [2.6, part (iii). As for (i), we claim that the sequence $\left(e_{n} / n^{1 / 2 p}\right)$ is in $l_{\text {weak }}^{2}(X)$ so that Proposition 2.6 applies. In case $p \geq 2$, we noted after Definition 2.5 that $\left(e_{n}\right)$ is in $l_{\text {weak }}^{2}(X)$ whence, $\left(x_{n}\right)=\left(e_{n} / n^{1 / 2 p}\right)$ is also; thus we can assume $p<2$. Let $x^{*}=\left(x_{n}^{\prime}\right)$ be in $X^{*}=l^{q}$. Then we have $x^{*}\left(x_{n}\right)=x_{n}^{\prime} / n^{1 / 2 p}$, and thus $S=\Sigma x^{*}\left(x_{n}\right)^{2}=\Sigma\left({x^{\prime}}_{n}^{2} / n^{1 / p}\right)$. Now as 
$q>2$, we can use $r=q / 2$ and $r^{*}=q /(q-2)$ to apply Holder's inequality, thus $S \leq\left[\Sigma\left|x_{n}^{\prime}\right|^{q}\right]^{2 / q}\left[\Sigma 1 / n^{q / p(q-2)}\right]^{1 / r^{*}}$. The series in the second $[\ldots]$ converges since $q>p(q-2)$, and so we get $S \leq\left\|x^{*}\right\|^{2} C_{1}$, where $C_{1}$ is equal to the sum of this last series and so the claim. Hence Proposition 4.3 is proved.

\subsection{Rademacher measures in ca and into $l^{p}$ with $p \geq 2$. Let}

$$
\mu(E):=\Sigma\left(\int_{E} r_{n}(t) d t\right) e_{n} .
$$

Analogous to the Trig measure by Proposition 2.6, this too has its values in $l^{p}$ (for $p \geq 2$ ) and is countably additive. Let us state without proof its corresponding properties. We note in part (ii) the contrast to the Trig measure in Proposition 3.5. if $p=2$, then the Rademacher measure's range contains an interior point (this result is due to Kluvanek [K-K]). Part (i) is in [A1].

Proposition 4.5 ( $\mathrm{A} 1] \mathrm{K}-\mathrm{K}])$. Let $X=l^{p}$ with $p \geq 2$. The Rademacher measure in (14) has the following properties:

(i) Its $T$ extends to $L^{p}$ for all finite $p>1$ but not to $L^{1}(A)$ on any set $A$ with $\lambda(A)>0$.

(ii) The set $C$ contains zero as an interior point iff $p=2$.

(iii) It is $N W F V$.

\section{Sum of COUntably Many SEgments}

A set $K$ in an arbitrary Banach space $X$ is called in [P-Ro] "the sum of countably many segments" if there exists an unconditionally convergent series $\Sigma x_{n}$ (in $X$ ) such that

$$
K:=\Sigma\left[-x_{n}, x_{n}\right] .
$$

Then $K$ is convex, and it is also norm compact thanks to a classical theorem due to Gelfand. The following elegant result is proved in [P-Ro]: A measure $\mu$ is in cca iff its range (i.e., its closed convex hull $K_{\mu}$ ) is contained in a sum of (countably many) segments. We observe that Proposition [5.1 strengthens a result in [R] and answers a question raised in $\mathrm{A}-\mathrm{D}$.

Proposition 5.1. If $\mu$ is in cca $(\lambda)$ and is $N W F V$, then there is a measure $\nu$ of $\sigma$-finite variation such that $K_{\mu} \subset K_{\nu}$.

Proof. By the result in [P-Ro, there exists an unconditionally convergent series $\Sigma x_{n}$ such that $K_{\mu} \subset K$ with $K$ in (15). But then using this series we may define first a $\sigma$-finite measure on Borel subsets of $[0,1]$ with range $K_{1}=\Sigma\left[0, x_{n}\right]$. Next we may define another such $\nu$ on Borel subsets of $[-1,1]$ with range $K_{\nu}=K$. Hence Proposition 5.1 is proved.

It is seen in $\mathrm{A}$-D that the sequence $\left(e_{n} / n\right)$ in $l^{1}$ does not lie in $K_{\mu}$ for any measure $\mu$ with finite variation. The question is raised if this sequence must always lie in range of a measure with non- $\sigma$ finite variation. As this sequence lies in range of measure of NWFV by Theorem 3.1 with $p=1$, the answer is no by Proposition 5.1 and we have the following.

Corollary 5.2. The sequence $\left(e_{n} / n\right)$ in $l^{1}$ lies in $K_{\mu}$ for some measure $\mu$ with $N W F V$ and also in (another) $K_{\nu}$ with $\sigma$-finite variation. 


\section{ACKNOWLEDGMENTS}

Part of the work for this paper was done when the author was visiting IIT Madras (Chennai, India). He wishes to thank Professor P. V Subrahmanyam of IIT Madras for kindly arranging the visit and for useful discussions.

\section{REFERENCES}

[A1] R. Anantharaman, Weakly null sequences in range of a vector measure and its integration map, Acta. Sci. Math. (Szeged) 70 (2004), 167-182. MR2072697 (2005d:28024)

[A2] R. Anantharaman, The sequence of Rademacher averages of measurable sets, Comment. Math. Prace Mat. 30 (1990), 5-8 (1991). MR1111780(92d:46070)

[A-D] R. Anantharaman and J. Diestel, Sequences in the range of a vector measure, Comment. Math. Prace Mat. 30 (1991), 221-235. MR1122692 (92g:46049)

[A-G] R. Anantharaman and K. M. Garg, The properties of a residual set of vector measures, in Measure Theory and Its Applications, Proc. Conf. Sherbrooke, Quebec (Canada), 1982, J.M. Belly, J. Dubois and P. Morales (eds.), Lecture Notes in Math. 1033, Springer-Verlag, Berlin, 1983, 12-35. MR729522 (86d:28014)

[D] J. Diestel, Sequences and Series in Banach Spaces, Graduate Texts in Math. 92, SpringerVerlag, New York, 1984. MR737004 (85i:46020)

[D-U] J. Diestel and J. J. Uhl, Jr., Vector measures, Math. Surveys 15, Amer. Math. Soc., Providence, RI, 1977. MR0453964 (56:12216)

[D-J-T] J. Diestel, H. Jarchow and A. Tonge, Absolutely summing operators, Cambridge Studies in Advanced Math. 43, Cambridge Univ. Press, 1995. MR1342297 (96i:46001)

[Dr-L] L. Drewnowski and Z. Lipecki, On vector measures which have everywhere infinite variation or noncompact range, Dissert. Math. (Rozprawy Mat.) 339, Warsawa, 1995. MR:1316274 (96f:46085)

[Du-S] N. Dunford and J. Schwartz, Linear Operators. I, Interscience, New York, 1958. MR0117523 (22:8302)

[J-K] L. Janicka and N. J. Kalton, Vector measures of infinite variation, Bull. Acad. Polon. Sci. Sér. Sci. Math. Astronom. Phys. 25 (1977), 239-241. MR0444889 (56:3235)

[K-S] B. Kacmarz, H. Steinhaus, Theorie der Orthogonale Reihen, Chelsea reprint, 1935.

[K-K] I. Kluvánek and G. Knowles, Vector measures and control systems, North-Holland, American Elsevier, Amsterdam-New York, 1976. MR0499068 (58:17033)

[L] J. Lindenstrauss, A short proof of Liapounoff's convexity theorem, J. Math. Mech. 15 (1966), 971-972. MR0207941 (34:7754)

[L-T] J. Lindenstrauss and L. Tzafriri, Classical Banach Spaces. I, Springer-Verlag, Berlin and New York, 1977. MR0500056 (58:17766)

[P-Ro] C. Piñerio and L. Rodriguez-Piazza, Banach spaces in which every compact lies inside the range of a vector measure, Proc. Amer. Math. Soc. 114 (1992)(2), 505-517. MR 1086342 (92e:46038)

[R] L. Rodriguez-Piazza, Derivability, variation and range of a vector measure, Studia Math. 112 (1995), 165-187. MR 1311694 (96c:28014)

[Ro] H. P. Rosenthal, On quasi-complemented subspaces of Banach spaces with an appendix on compactness of operators from $L^{p}(\mu)$ to $L^{r}(\nu)$, J. Funct. Anal. 4 (1969), 176-214. MR0250036 (40:3277)

[T] E. Thomas, The Lebesgue-Nikodym theorem for vector valued measures, Mem. Amer. Math. Soc. 139 (1974).

[Z] A. Zygmund, Trigonometric Series, 2nd ed., Vols. I, II, Cambridge Univ. Press, New York, 1959. MR0107776(21:6498)

Professor Emeritus, Department of Mathematics and Computer information Sciences, Suny College at Old Westbury, Old Westbury, New York 11568-0210

E-mail address: rajan_a2000@yahoo.com 